\title{
DIELECTRIC STUDY OF PURE POLYMER AND MIXED MONOMER-POLYMER SINGLE CRYSTALS OF pTS DIACETYLENE: A COMPARISON WITH NEUTRON SCATTERING RESULTS
}

\author{
S. Longeville, J. Even, M. Bertault
}

Groupe Matière Condensée et Matériaux, URA au C.N.R.S. 804, Université de Rennes I Campus de Beaulieu, 35042 Rennes Cedex, France

\author{
H.A. KoLODZIEJ \\ Institute of Chemistry, University of Wrocław \\ F. Joliot-Curie 14, 50-383 Wrocław, Poland
}

AND J. SWORAKOWSKI

Institute of Physical and Theoretical Chemistry, Technical University of Wrocław Wybrzeze Wyspiańskiego 27, 50-370 Wrocław, Poland

(Received April 25, 1995)

Dedicaled to Professors Krzysztof Pigoń, Józef W. Rohleder and Zdzistaw Ruziewicz on the occasion of their 70th birthdays*

Temperature and frequency dependences of the electric permittivity in mixed monomer-polymer single crystals of the diacetylene pTS (2,4-hexadiynylene bis ( $p$-toluenesulphonate)) are reported. The dependences were determined in the temperature range 100-250 K, and in the frequency range $100 \mathrm{~Hz}-1 \mathrm{MHz}$. Two types of single crystals were studied: nearly-fully polymerized ones, and those containing ca. $20 \%$ of polymer in the monomer matrix. The dynamics is characterized by a high-frequency response $(\nu \gg 3$ $\mathrm{GHz}$ ) which corresponds to the soft mode analysed previously by the Raman and neutron inelástic scatterings. At low frequencies $(\nu \leq 1 \mathrm{kHz})$, another component is observed, its dielectric intensity peaking around the transition temperature in the fully polymerized crystals. The results are consistent with the results obtained from NMR and neutron scattering experiments.

PACS numbers: $64.70 . \mathrm{Kb}, 77.84 . \mathrm{Jd}, 61.12 .-\mathrm{q}$

* Submitted on invitation of the Institute of Physical and Theoretical Chemistry, Technical University of Wrocław, Wroclaw, Poland. 


\section{Introduction}

Several diacetylenes $\left(\mathbf{R}_{1}-\mathrm{C} \equiv \mathrm{C}-\mathrm{C} \equiv \mathrm{C}-\mathrm{R}_{2}\right)$ are reactive in the solid state (see, e.g., [1-3] for reviews and collections of references). Among them, the model diacetylene is 2,4-hexadiynylene-1,6-bis( $p$-toluenesulfonate); the molecule, hereafter referred to as pTS, contains two identical, highly polar $p$-toluenesulfonate side groups $\left(\mathrm{R}_{1}=\mathrm{R}_{2}=-\mathrm{O}-\mathrm{SO}_{2}-\mathrm{C}_{6} \mathrm{H}_{4}-\mathrm{CH}_{3}\right)$. Solid pTS undergoes a single crystal-single crystal topochemical polymerization upon exposure to radiation (UV, $\gamma, \mathrm{X}, \ldots$ ) or by thermal annealing [4]. The kinetics of thermal polymerization of pTS and its fully deuterated analogue (hereafter pTS-H and pTS-D, respectively) is well known; it is thus possible to obtain mixed monomer-polymer $\left(\mathrm{M}_{1-x} \mathrm{P}_{x}\right)$ single crystals with any polymer contents $(x)$ [5].

Both monomer and fully polymerized $\mathrm{pTS}-\mathrm{H}$ and $\mathrm{pTS}-\mathrm{D}$ are isostructural [5-10]. In the fully polymerized crystals, an antiferroelectric phase transition takes place at $T_{\mathrm{c}}=190 \mathrm{~K}$ [10-12]. The transition is associated with freezing of librational motions of the polar side groups, yielding two inequivalent sites for each structural unit and resulting in a doubling of the unit cell along the $a$ crystallographic axis. A soft mode has been observed in the high-temperature phase by neutron scattering, and in the low-temperature phase by Raman scattering [10]. In addition, a central peak related to defects (monomer units in the polymer lattice) was found to build up near $T_{\mathrm{c}}$. In crystals of the pTS-H and pTS-D monomers, an intermediate incommensurate phase is present [13-15]. The soft mode has the same mean-field behaviour in monomer and polymer crystals. A central peak is also observed in monomer.

In mixed monomer-polymer crystals [16], a medium-range ordering (MRO) is stable at low temperatures $\left(T \leq T^{\mathrm{MRO}}\right)$. The value of $T^{\mathrm{MRO}}$ depends on the polymerization degree, amounting to $c a .180 \mathrm{~K}$ in fully polymerized crystals and decreasing to $c a .80 \mathrm{~K}$ in mixed crystals in which $x \approx 0.4-0.6$. Above this temperature, in the $T^{\mathrm{MRO}}-T_{\mathrm{c}}$ range, a local ordering is observed along the $b$ axis, which is the direction of the stacks of monomer molecules and of the growing polymer chains. The soft mode in the mixed crystals has almost the same behaviour as that in both pure monomer and pure polymer except for a finite frequency gap at $T_{\mathrm{c}}$. The order parameter fluctuation spectrum is dominated by a very strong central peak, responsible for the local ordering in the $T^{\mathrm{MRO}}-190 \mathrm{~K}$ temperature range.

The central peak contribution appeared static in our neutron scattering experiments. If one postulates that this central peak has a dynamical origin and corresponds to a typical frequency $\nu_{\mathrm{p}}$, then $\nu_{\mathrm{p}}$ should be smaller than the neutron energy resolution: $\nu_{\mathrm{p}} \ll 10 \mathrm{GHz}$. The doubling of the $a$ parameter of the unit cell was also observed by NMR performed on deuterated samples in both mixed monomer-polymer and almost fully polymerized crystals at ca. $190 \mathrm{~K}$ [10]. We conclude from this result that $\nu_{\mathrm{p}}$ should not exceed the Larmor frequency $\left(\nu_{\mathrm{p}} \ll 50 \mathrm{MHz}\right)$.

In our earlier papers $[12,17]$ we presented results of the study of polymerization and phase transitions in crystals of pTS-H employing the dielectric measurements. The shape, position and magnitude of the anomaly associated with the phase transition was found dependent on the polymer contents. In particular, we 
reported on the temperature and frequency dependences of the electric permittivity measured in fully polymerized crystals along the $b$ direction in the $150-270 \mathrm{~K}$ and $1 \mathrm{kHz}-2.8 \mathrm{GHz}$ ranges [12]; no dispersion was observed in these measurements. However, as the soft mode is important, the soft mode scattering cross-section is reduced near $T_{\mathrm{c}}$ in both polymer and monomer $[10,18]$ to a signature equivalent to a relaxation process. In such a case, a dispersion should be observed [19].

In the present paper we report on results of a re-investigation of the electric permittivity of pTS. The range of the experiments was extended towards lower frequencies $(100 \mathrm{~Hz})$ and lower temperatures $(100 \mathrm{~K})$. The measurements were carried out on fully polymerized crystals, and on a mixed $M_{1-x} \mathrm{P}_{x}$ system $(x \approx 0.2)$; the latter system was chosen because the neutron experiments [16] demonstrated that the disorder reaches its maximum for compositions in which the incommensurate phase disappears.

\section{Experimental and results}

Crystals of pTS-H were obtained using the method described in detail elsewhere [20]. The crystals of monomer were grown by a slow evaporation of the acetone solution of pTS at $277 \mathrm{~K}$, under a controlled flow of nitrogen.

As was determined in our earlier experiments $[12,17]$, anomalies of the electric permittivity associated with the phase transitions are best observed on the $\varepsilon_{2}$ component of the permittivity tensor. Thus the samples used in the measurements reported in this paper were cut from as-grown crystals perpendicular to the $b$ direction; their thicknesses amounted typically to $1 \mathrm{~mm}$, their electroded areas to $5 \times 3 \mathrm{~mm}^{2}$. Partly polymerized crystals containing a pre-determined fraction of polymer were obtained by thermal annealing of samples at $333 \mathrm{~K}$ during a time deduced from the isothermal polymerization kinetics of small pieces of the same crystals at the same temperature.

The electric permittivities were measured in the frequency range from $100 \mathrm{~Hz}$ to $1 \mathrm{MHz}$ using an IIP-5284A precision LCR meter. The crystals were mounted in a special sample holder $[21,22]$ cooled with liquid nitrogen which passed through a heat exchanger provided with the heater and a temperature sensor connected to a PDI temperature controller. The sample temperature was measured with a copper-constantan thermocouple to within $\pm 0.1 \mathrm{~K}$. The experiments covered the temperature range $100-260 \mathrm{~K}$.

Figure 1 shows the results of the measurements of electric permittivity in a fully polymerized pTS-H sample $(x \approx 1)$, as well as in a mixed crystal $\mathrm{M}_{0.8} \mathrm{P}_{0.2}$. The $\varepsilon^{\prime}(T)$ dependences for pure polymer, shown in Fig. 1a, exhibit a maximum around $T_{\mathrm{c}}=190 \mathrm{~K}$, in a good agreement with previous dielectric $[12,17,23]$, and Raman and neutron scattering $[10,11]$ experiments. The magnitude of the anomaly associated with the transition decreases with increasing frequency. It should also be noted that $\varepsilon^{\prime}$ is an increasing function of frequency below the transition $(T<160 \mathrm{~K})$, whereas at higher temperatures $\varepsilon^{\prime}$ decreases as the frequency increases, becoming finally nearly frequency-independent above $c a .230 \mathrm{~K}$. At high frequencies, our results are consistent with the previously published ones $[12,17,23]$ which predict the ratio of the slopes of the $\left(\varepsilon^{\prime}\right)^{-1}(T)$ dependences be- 

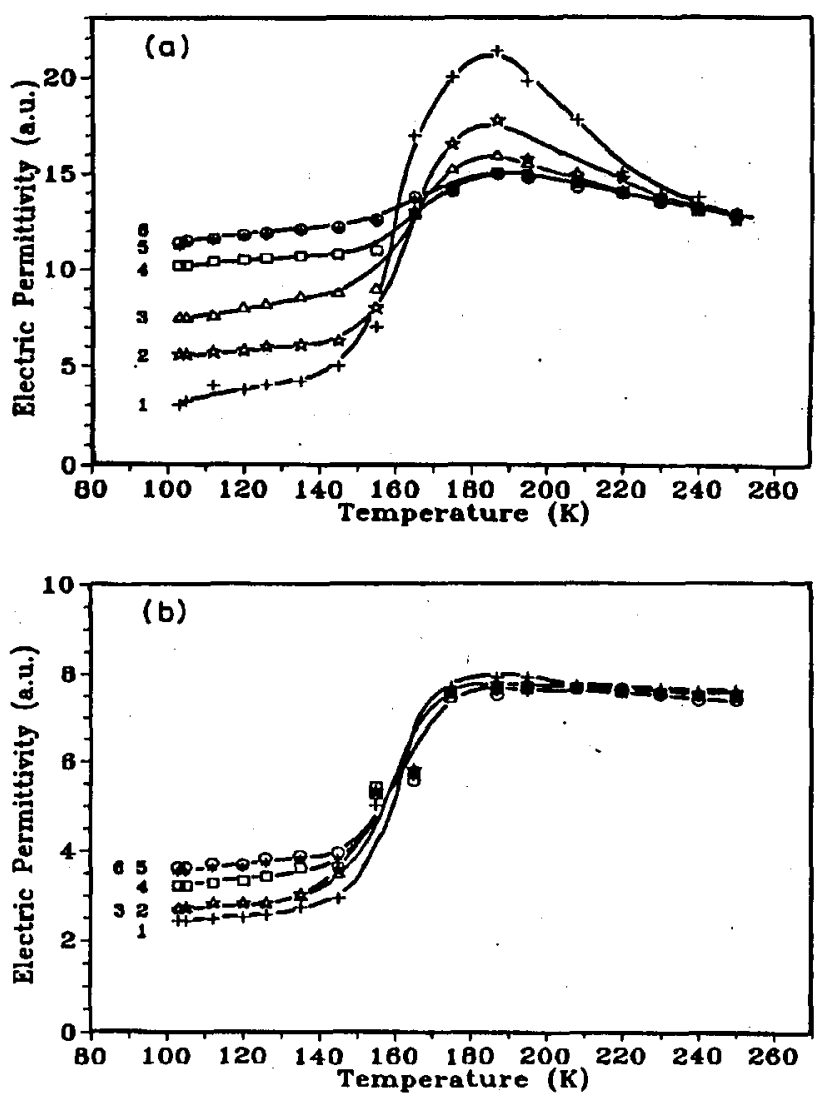

Fig. 1. Temperature dependences of the real part of the relative electric permittivities measured along the $b$ crystallographic direction in a fully polymerized pTS crystal (a) and in a mixed $M_{0.8} P_{0.2}$ crystal (b). The measurement frequencies amount to $100 \mathrm{~Hz}$ (1), $500 \mathrm{~Hz}(2), 1 \mathrm{kHz}(3), 5 \mathrm{kHz}(4), 50 \mathrm{kHz}(5)$, and $1 \mathrm{MHz}$ (6). The permittivity scale is given in arbitrary units, 1 a.u. being approximately equal to $0.5 \varepsilon$ unit.

low and above the transition to amount to -2 . At the lowest frequencies covered by the present experiments, this ratio is higher.

A much weaker temperature dependence was found in the partly polymerized crystal (cf. Fig. 1b). Irrespective of the measurement frequency, we have not observed any sharp maximum: on decreasing the temperature, we recorded almost constant (or very weakly increasing) values of $\varepsilon^{\prime}$ down to $180-190 \mathrm{~K}$, then the permittivities decreased to reach new almost constant values below ca. $120 \mathrm{~K}$. Unlike the result obtained for fully polymerized crystals, $\varepsilon^{\prime}$ was never a decreasing function of frequency. We must also point out that the dispersion such as that expected of order-disorder phase transitions [19] has never been observed in our experiments performed on monomer-polymer mixed crystals. 


\section{Discussion}

Earlier experiments performed on PTS-D [10] have shown that the fluctuation spectrum contains two types of signatures: one, with a characteristic frequency lower than $10 \mathrm{MHz}$, leads to the experimental central peak observed by the neutron scattering, the other one is a soft mode $\left(\omega_{\infty} \approx 250 \mathrm{GHz}\right.$ at $\left.220 \mathrm{~K}\right)$. Both signatures are critical at $T_{\mathrm{c}}$ : the soft mode decreases to almost zero and the central peak intensity diverges. The soft mode is overdamped in the high-temperature phase (above $T_{\mathrm{c}}$ ). Below $T_{\mathrm{c}}$, three components are observed: a static Bragg peak (independent of the wave vector $q$ ), a central peak with a $\boldsymbol{q}$ dispersion, and a soft mode. Near $T \approx 160 \mathrm{~K}$, the soft mode suddenly recovers a small damping and a high frequency. Simultaneously, almost the entire intensity of the central peak is transferred to the Bragg peak. The dynamic character of the central peak in mixed monomer-polymer crystals of pTS has not been experimentally confirmed to date.

In the analysis of the results presented in this paper, we shall employ a model, used previously to explain features of inelastic neutron and Raman scattering data [10]: the presence of two signatures will be assumed, one with a resonant character (the soft mode), the other - with a relaxational character (the central peak). This approach is similar to that used by Shapiro et al. [24] to describe the fluctuation spectrum in crystals with the perovskite structure. Within the model, the soft-mode susceptibility can be described by coupling of an oscillator to a relaxator

$$
\chi^{-1}(\omega)=\omega_{\mathrm{c}}^{2}-\omega^{2}-\mathrm{i} \omega\left(\Gamma+\frac{\delta^{2}}{\gamma-\mathrm{i} \omega}\right)
$$

where $\omega_{\mathrm{c}}$ is the soft mode frequency (not renormalized), $\Gamma$ and $\gamma$ are the damping constants of the oscillator and relaxator $(\gamma$ being related to the half-width (HWHM) of the central peak), and $\delta$ is the coupling coefficient. The above expression was successfully used [10] for partly and nearly-completely polymerized pTS-D $(x=0.4, x=0.8$, and $x=0.95)$; the values of $\omega_{\mathrm{c}}^{2}$ and $\delta$ were determined but only an upper limit was obtained for $\gamma(\gamma \ll 10 \mathrm{GHz})$. The results reported in the present paper do not allow either for a quantitative determination of the coupling term $\delta$ or the characteristic frequencies $\omega_{\mathrm{c}}$ and $\gamma$. We shall, nevertheless, verify the compatibility between the results obtained from the inelastic neutron and Raman scattering, and those obtained from the frequency and temperature dependence of the real part of the electric permittivity reported in this paper.

As is shown in Fig. 1a, in the high-temperature phase $\left(T>T_{\mathfrak{c}}\right)$ the electric permittivity in the fully polymerized pTS crystal is a decreasing function of frequency; such a behaviour is consistent with the presence of a dynamical central peak and an overdamped soft mode. The behaviour in the vicinity of the transition is related to the critical signatures of both the soft mode and the central peak. The anomaly at $T=T_{\mathrm{c}}$ is mainly due to the central peak [10], whose contribution influences the frequency dependence of the permittivity. Such a dependence, shown in Fig. 2, demonstrates that the central peak has a maximum contribution below $100 \mathrm{~Hz}$ but its influence is non-negligible up to $c a .10 \mathrm{kHz}$. For $T<160 \mathrm{~K}$, the values of $\varepsilon^{\prime}$ are lower than those in the high-temperature phase. The soft mode in 


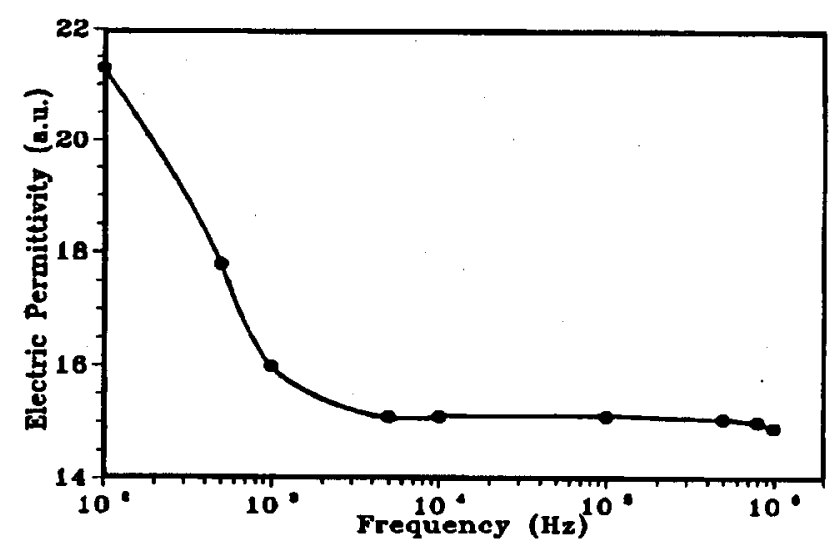

Fig. 2. Frequency dependence of the real part of the relative electric permittivity in fully polymerized pTS-H crystal, measured at $T=T_{\mathrm{c}}$.

this temperature range is no more overdamped, and its frequency rapidly increases [2]. The characteristic frequency of the central peak decreases below $T_{\mathrm{c}}$ and then probably becomes of a static nature [2]. There is therefore a smaller contribution of the central peak in the low-frequency range. The frequency dependence of the real part of the dielectric susceptibility, deduced from the above discussion, is schematically shown in Fig. 3. For comparison, we also show the differential scattering cross-section $S(q, \omega)$ determined by the inelastic neutron scattering (Fig. 4), which, within the frame of the fluctuation-dissipation theorem [25], is related to the imaginary part of the susceptibility

$$
S(q, \omega)=[n(\omega)+1] \chi^{\prime \prime}(q, \omega),
$$

$n(\omega)$ being the phonon occupation number.

In the mixed $\mathrm{M}_{0.8} \mathrm{P}_{0.2}$ pTS crystal, the evolution of the real part of the dielectric susceptibility (and hence $\varepsilon^{\prime}$ ) with temperature is slightly different. The differences can be related to differences in the behaviour of both the central peak and the soft mode in the mixed crystals, in which the transition appears more diffuse than in pure monomer or pure polymer crystals [16]. The intensity of the central peak continuously increases from high temperatures down to $10 \mathrm{~K}$. On the other hand, the soft mode exhibits a smaller variation in the high-temperature phase, presenting at $T=T_{\mathrm{c}}$ a gap more important than in pure polymer. Therefore, the part of the susceptibility associated with the soft mode has less critical character at $T_{c}$, and the behaviour of the central peak is also less critical, the contribution of the latter factor being much more important. The results of the dielectric measurements are consistent with these findings: as is shown in Fig. 1b, the permittivity above $c a .170 \mathrm{~K}$ is almost temperature-independent whatever the frequency, exhibiting no critical behaviour at $190 \mathrm{~K}$. The evolution of the susceptibility in this temperature range is related to the evolution of the central peak, and probably does not contain any appreciable contribution from the soft mode. Between $c a .170 \mathrm{~K}$ and $c a .150 \mathrm{~K}$, the permittivities decrease, the magnitude of the effect being more prominent for low frequencies, and almost negligible above 

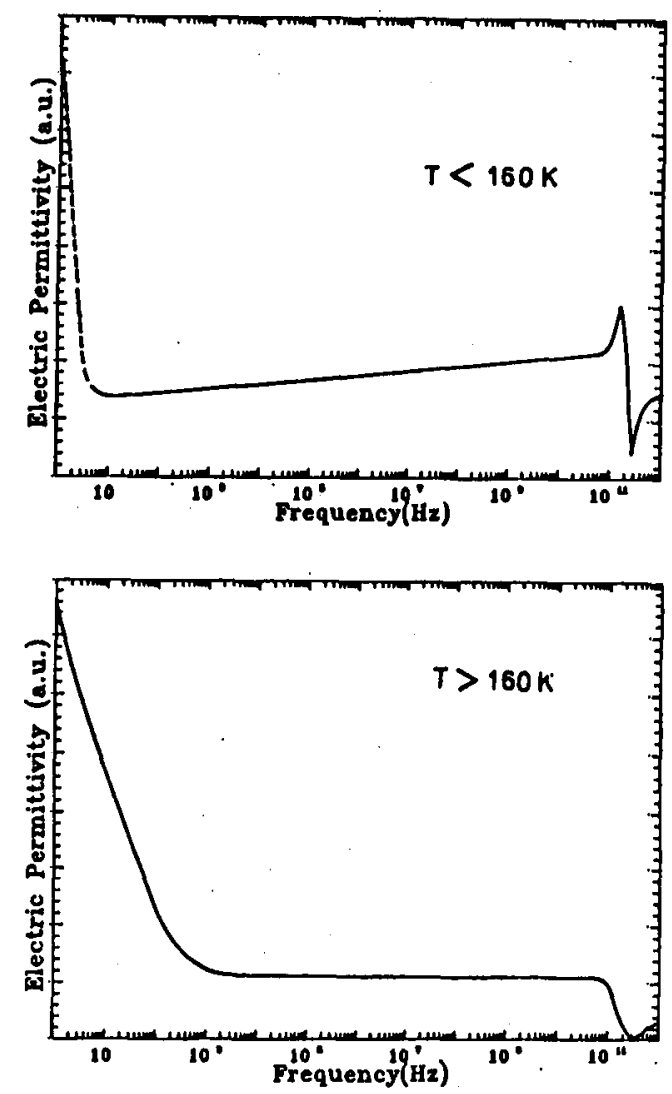

Fig. 3. A scheme of the frequency dependence of the real part of the electric permittivity in pTS polymer above and below $160 \mathrm{~K}$. See text for further discussion.

$5 \mathrm{kHz}$. This effect, similar to that observed in pure polymer crystals (cf. Fig. 1), can probably be explained in the same way, by a decrease in the contribution from the central peak at low frequencies. The decrease in the permittivity between the high- and low-temperature phases is probably not related to the increase in the soft mode frequency because only a small variation of $\varepsilon^{\prime}$ is observed between $5 \mathrm{kHz}$ and $1 \mathrm{MHz}$.

The dielectric measurements reported in this paper yield results consistent with those obtained by other techniques: at high temperatures, the response is due to the existence in the fluctuation spectrum of a central peak and a soft mode, whereas it depends only on the presence of the soft mode in the low-temperature phase. In the partly polymerized $\mathrm{M}_{0.8} \mathrm{P}_{0.2}$ crystal, the soft mode contribution is less important than in the pure polymer. An anomaly appears at very low frequencies in the fluctuation spectrum of the order parameter. At $T<160 \mathrm{~K}$, this signal is related to the random field induced by polymer chains in the monomer matrix. The contribution of the central peak is observed in the dielectric measurements in the low-frequency range $(100 \mathrm{~Hz}-5 \mathrm{kHz})$ at $T>160 \mathrm{~K}$, disappearing at lower 

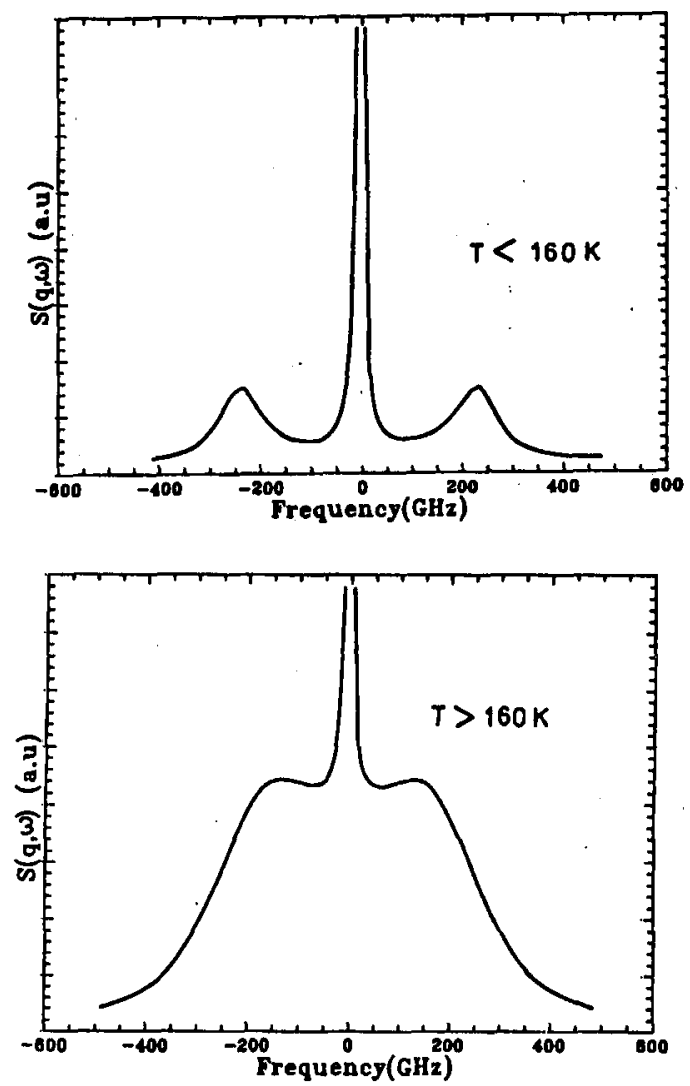

Fig. 4. The frequency dependence of the scattering cross-section measured by inelastic neutron scattering above and below $160 \mathrm{~K}$. The soft mode is overdamped at $T>160 \mathrm{~K}$, and underdamped at $T<160 \mathrm{~K}$. The HWHM of the central peak response is given by the resolution function (ca. $40 \mathrm{GHz})$.

temperatures. This is consistent with neutron scattering experiments which show a Bragg-like peak below ca. $160 \mathrm{~K}$. The latter temperature also corresponds to the temperature of the lock-in transition in pure monomer. These findings indicate that the disorder in mixed crystals is probably related to small differences between the critical wave vector of the monomer $q_{s}=0.5 a^{*} \pm 0.06 b^{*}$, and the critical wave vector of the polymer $q_{c}=0.5 a^{*}$ [26]. In the mixed crystals, the anomaly of $\varepsilon^{\prime}$. is related to the decrease in the characteristic frequency of the central peak, and should therefore appear a few tens of Kelvins below the transition temperature. This could account for the differences in the transition temperatures determined from the dielectric measurements [12] and from other techniques [27, 28].

\section{References}

[1] Polydiacetylenes, Ed. H.-J. Cantow, Springer Verlag, Berlin 1984.

[2] Polydiacetylenes: Synthesis, Structure and Electronic Properties, Eds. D. Bloor, R.R. Chance, Nijhoff Publ., Dordrecht 1985. 
[3] M. Schott, G. Wegner, in: Nonlinear Optical Properties' of Organic Molecules and Crystals, Vol. 2, Eds. D.S. Chemla, J. Zyss, Academic Press, New York 1987, p. 3.

[4] G. Wegner, Z. Nat.forsch. B 24, 824 (1969).

[5] M. Bertault, M. Schott, M.J. Brienne, A. Collet, Chem. Phys. 85, 481 (1984).

[6] D. Kobelt, E.F. Paulus, Acta Crystallogr. B 30, 232 (1974).

[7] V. Enkelmann, Acta Crystallogr. B 33, 2842 (1977).

[8] V. Enkelmann, G. Wegner, Angew. Chem. Int. Ed. 16, 416 (1977).

[9] J.P. Aimé, J. Lefebvre, M. Bertault, M. Schott, J.O. Williams, J. Phys. (France) 43, 307 (1982).

[10] J. Even, M. Bertault, B. Toudic, H. Cailleau, J.L. Fave, R. Currat, F. Moussa, Phys. Rev. B 49, 11602 (1994).

[11] D. Bloor, D.A. Fisher, D.N. Batchelder, R. Kennedy, A.C. Cottle, W.F. Lewis, M.B. Hursthouse, Mol. Cryst. Liq. Cryst. 52, 83 (1979).

[12] R. Nowak, J. Sworakowski, B. Kuchta, M. Bertault, M. Schott, R. Jakubas, H.A. Kołodziej, Chem. Phys. 104, 467 (1986).

[13] P. Robin, J.P. Pouget, R. Comes, A. Moradpour, J. Phys. (France) 41, 415 (1980).

[14] J. Even, B. Toudic, M. Bertault, H. Cailleau, F. Moussa, to be published.

[15] M. Bertault, J. Even, Chem. Mater. 6, 1343 (1994).

[16] S. Longeville, J. Even, M. Bertault, F. Moussa, to be published.

[17] M.E. Orczyk, Chem. Phys. 142, 485 (1990).

[18] J. Even, H. Cailleau, B. Toudic, M. Bertault, F. Moussa, R. Currat, Physica B 180, 339 (1992).

[19] R. Blinc, B. Žekš, Soft Modes in Ferroelectrics and Antiferroelectrics, North-Holland, Amsterdam 1974.

[20] M. Bertault, Ph.D. Thesis, Université Paris VII, Paris 1983.

[21] H.A. Kołodziej, L. Sobczyk, Acta Phys. Pol. A 39, 59 (1971).

[22] P. Freundlich, H.A. Kołodziej, E. Narewski, J. Phys. E, Sci. Instrum. 14, 1045 (1981).

[23] R. Zieliński, J. Kalinowski, J. Phys. C, Solid State Phys. 20, 177 (1987).

[24] S.M. Shapiro, J.D. Axe, G. Shirane, T. Riste, Phys. Rev. B 6, 4332 (1972).

[25] B. Dorner, R. Comes, in: Dynamics of Solids and Liquids by Neutron Scattering, Topics in Current Physics No. 3, Ed. S.W. Lovesey, Springer, Berlin 1977, p. 127.

[26] A.P. Levanyuk, private communication.

[27] J.N. Patillon, P. Robin, P.A. Albouy, J.P. Pouget, R. Comes, Mol. Cryst. Liq. Cryst. 76, 297 (1981).

[28] J.P. Aimé, M. Bara, M. Bertault, M. Schott, Mater. Sci. 10, 441 (1984). 